\title{
Humanización
}

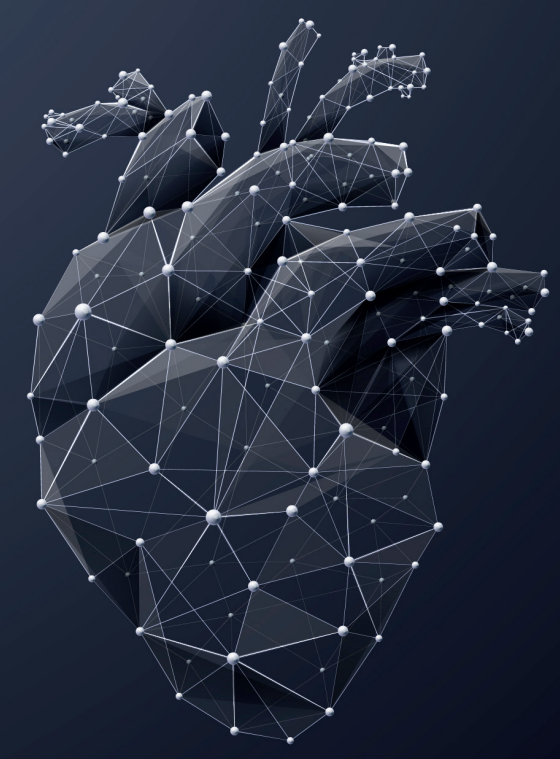

\section{La humanización de la salud y las nuevas tecnologías}

The Humanization in Health and the New Technologies 
Resumen

Abstract
El advenimiento de los avances cientificos y tecnológicos aportó a la medicina una importante variedad de recursos; esto permitió a los profesionales de la salud mejorar la calidad asistencial en cuanto a diagnóstico y terapéutica. Pero, por otro lado, en ocasiones su uso repercutió desfavorablemente en la relación paciente-equipo de salud.

El presente trabajo tiene por objetivo evaluar retrospectivamente la implementación de las nuevas tecnologías y su impacto sobre esta relación, reflexionando desde la perspectiva bioética, la responsabilidad que les cabe en el proceso de deshumanización de la medicina.

The emergence of scientific and technological advances contributed an important variety of resources to medicine; this allowed health professionals to improve the quality of care, in terms of diagnostic and therapy. But on the other hand, on occasions their use rebounded unfavorably in the patienthealth team relationship.

The present work has the objective of retrospectively evaluating the implementation of new technologies and their impact on this relationship, reflecting from the perspective of bioethics, the responsibility that attributed to them in the de-humanization of medicine process.

Key words Humanización; paciente; equipo de salud; nuevas tecnologías.

Humanization; patient; health team; new technologies.

Recibido: 24/04/2018. Aceptado: 29/08/2018 


\section{Introducción}

El advenimiento de los avances científicos y tecnológicos aportó a la medicina asistencial una importante variedad de recursos que permitió a los profesionales de la salud mejorar la calidad asistencial en cuanto a diagnóstico y terapéutica.

De este modo, dichos adelantos facilitaron la posibilidad de concretar diagnósticos más precisos en menor tiempo, concediendo la oportunidad de brindar tratamientos específicos, mejorando la calidad y la expectativa de vida.

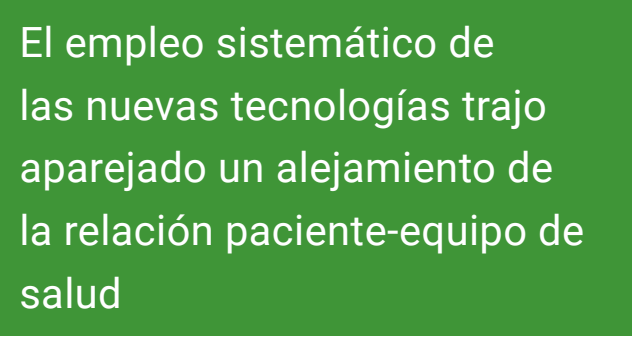

Pero, por otro lado, en ocasiones debido a su inapropiado manejo se transformaron en un obstáculo en la relación paciente-equipo de salud, fundamentada en la empatía entre seres humanos.

El empleo sistemático de las nuevas tecnologías trajo aparejado un alejamiento de esta relación; algunos profesionales utilizan el mayor tiempo de la consulta en solicitar exámenes de diagnóstico complementarios o en evaluar sus resultados, en desmedro de la comunicación con la persona o preocupándose más por operar un equipo que por las necesidades del enfermo.

El presente trabajo tiene por objetivo evaluar retrospectivamente la implementación de algunas de las nuevas tecnologías y su impacto sobre la relación paciente-equipo de salud, reflexionando, desde la perspectiva bioética, la responsabilidad que tienen en el proceso de deshumanización de la medicina.

En este contexto cabe citar que ciertas instituciones, como la Orden Hospitalaria de San Juan de Dios ${ }^{1}$ aportan desde su origen un valor agregado a la humanización de la salud, manifestando su preocupación por el tema y sosteniendo acciones a favor de la misma, en los centros donde se lleva adelante su misión.

\section{Humanización de la salud}

Para la Organización Mundial de la Salud, el concepto de salud se refiere al completo estado de bienestar físico, psíquico, mental y social y, no solamente a la mera ausencia de enfermedad (https://concepto.de/salud-segun-la-oms/).

Posteriormente, la Organización Panamericana de la Salud aportó a esta definición, sumando que también tiene que ver con la preservación del medio ambiente que rodea a la persona.

Este nuevo concepto reemplaza a la anterior acepción que consideraba solamente la esfera física, develando de este modo que la figura de la persona humana comprende varias dimensiones en sí misma, es pluridimensional.

1 San Juan de Dios (Montemor o Novo, Portugal, 1495 - Granada 8/3/1550) "Fundador de la Orden Hospitalaria. Iniciador del hospital moderno, padre de los pobres, ícono de caridad, profeta de la hospitalidad". (Matínez Gil, J. L. OH-2010.) 
La persona es un ser único y singular que se construye desde el seno materno por un proceso de ontogénesis $y$, en este complejo desarrollo que se sostiene durante toda la vida, intervienen factores genéticos, históricos y culturales (Ordi, 2011).

La enfermedad es una circunstancia que provoca la desestructuración de la persona, la atraviesa en cualquier momento de su existencia y la vulnera en su ser más íntimo; nunca es sectorial y, por lo tanto, requiere del especial cuidado en todas las dimensiones que la constituyen.

Cuando por dicha circunstancia se requiere de un cuidado mayor al que se brinda ambulatoriamente, la hospitalización obliga a interrumpir la rutina de vida, las relaciones personales, familiares, laborales; trasladarse a otro sitio ajeno a su hogar, provocando con esta situación miedo, angustia, preocupaciones e incertidumbre.

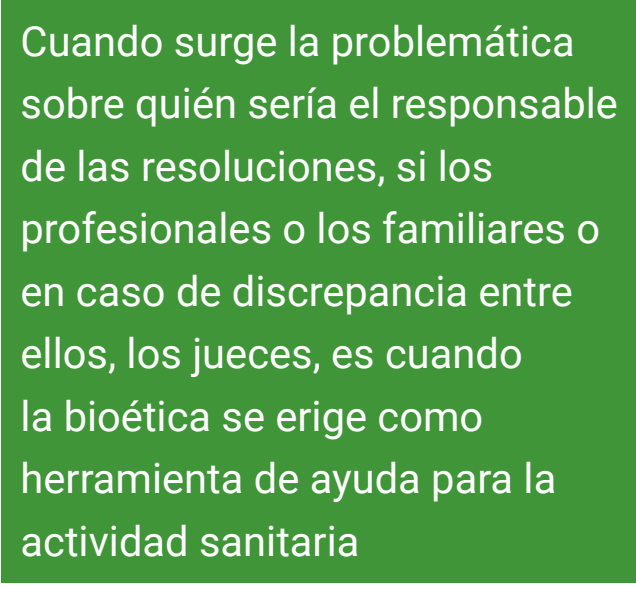

Cabe recordar que la medicina del siglo pasado se cimentaba, en las primeras décadas, en una relación paternalista médicopaciente; el profesional decidía qué era lo más adecuado para el enfermo y este, respaldado por la confianza que le brindaba su galeno, aceptaba sin objeciones las propuestas diagnósticas y terapéuticas.

No se tomaban en cuenta las preferencias ni las creencias de las personas, ni se solicitaba su consentimiento, del mismo modo, no se estimulaba la elaboración de líneas directrices anticipadas sobre sus deseos particulares en caso de patologías con criterios de terminalidad, conocidas hoy día como testamento vital.

Hacia la segunda mitad de la centuria, se produce un cambio en el modelo de la citada relación, virando la misma hacia una propuesta contractual, donde el agente sanitario propone y el enfermo dispone tomando las decisiones libremente, sin coerción externa y haciendo uso del derecho a expresar su voluntad autónoma (se refiere a un contrato de servicio entre dos partes y no a una relación mercantil).

El dilema afloró particularmente cuando las personas no podían hacer uso de este derecho, cuando por causa de la enfermedad de base, no podían expresar sus deseos, por encontrarse su autonomía limitada o abolida y derivada en terceros.

De este modo surge la problemática sobre quién sería el responsable de las resoluciones, si los profesionales o los familiares o en caso de discrepancia entre ellos, los jueces.

Fue entonces el momento oportuno para el surgimiento de la bioética, disciplina que se erige como herramienta de ayuda indispensable para la actividad sanitaria; conformándose así los comités de ética asistencial (CEA) y promoviendo en su seno el diálogo interdisciplinar, argumentando en el equipo las opciones posibles y confrontándolas con los principios y reglas morales, para arribar a una recomendación consensuada. 


\section{Factores que promueven la humanización en la asistencia sanitaria}

La humanización de la salud requiere de un proceso primario individual de los agentes de salud, para luego poder trasladar un trato humanizado al enfermo y, en las instituciones sanitarias esto se logra teniendo en cuenta varias líneas de acción.

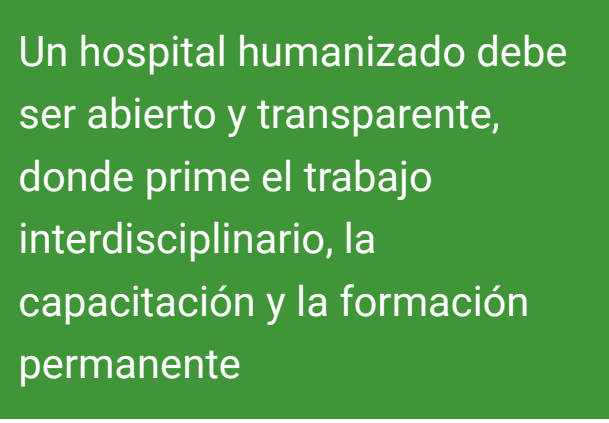

Una de ellas se alcanza a través de las políticas de recursos humanos, manteniendo un constante y sostenido trabajo en equipo con el pertinente profesionalismo de todos los trabajadores sanitarios.

Es primordial ubicar a la persona que sufre en el centro del interés de todos para lograr su bienestar; reponiendo su salud integral cuando sea posible o paliando los males que le aquejan, pero siempre cuidando al hombre desde un enfoque holístico, respetando su dignidad como persona.

La aparatología debe estar al servicio del hombre y no al revés; no hay que perder de vista a la persona que requiere de asistencia instrumentalizada para sostener su vida, cuidando todos los aspectos que la definen como tal: biológico, psíquico, moral, social y espiritual.

Son los trabajadores de la salud los responsables de que estas premisas se cumplan; esto permitirá un vínculo empático entre los profesionales y la persona asistida mediante una comunión intelectual, afectiva y espiritual.

A nivel institucional, un hospital humanizado debe ser abierto y transparente, donde prime el trabajo interdisciplinario, la capacitación y la formación permanente; esencialmente donde se respete al enfermo en todos sus derechos, brindando acogida y hospitalidad.

El proceso de humanización en los centros de salud obliga a ser dinámico e interactivo y las acciones tendientes a lograrlo deben ir dirigidas a los tres partícipes necesarios: la persona del enfermo y su entorno, los agentes de salud que allí se desempeñan y las directrices organizativas y estructurales. (Brusco, 1988²)

En palabras de Pier Luigi Marchesi, $\mathrm{OH}^{3}$ :

Un hospital que cura al enfermo pero que no se preocupa de su persona, corre el peligro de hacerse inhumano y deshumanizante, en el sentido más amplio de la palabra.

2 Pbro. Ángelo Brusco, religioso camilo, director del Centro Camiliano de Formación en Verona, Italia. Filósofo, teólogo y psicólogo.

3 Fr. Pier Luigi Marchesi (1929-2000), superior general de la Orden Hospitalaria de San Juan de Dios (1976-1988), considerado uno de los "grandes generales" de la orden por sus aportes. 


\section{Factores que provocan deshumanización en la asistencia sanitaria}

La cultura sanitaria deshumaniza cuando:

- La acogida es fría e impersonal.

- La asistencia solo se dedica a la enfermedad orgánica.

- No se preserva el secreto médico.

- No se respeta la privacidad.

- Se brinda información limitada o no se brinda información alguna.

- No se permite la participación del paciente en la toma de decisiones.

- Por lo citado, se evidencia que no se respeta el principio de autonomía, ni las reglas morales de intimidad, confidencialidad y veracidad cuando no se brinda información fidedigna y clara sobre el diagnóstico y el pronóstico.

Del mismo modo se afecta la cualidad humana cuando:

- Se tecnifican los problemas vitales del enfermo.

- Es excesiva la instrumentalización de la medicina.

- El profesional de la salud oficia como un mero técnico.

- La persona que sufre se transforma en un número o un caso clínico, sintiéndose como un objeto.

- Se dificulta la oportunidad de enriquecer el encuentro interpersonal entre los agentes de salud y el paciente, tan especial.

- Se provoca la segregación de enfermos que sufren patologías crónicas progresivas e invalidantes, no plausibles de curación; cuando la medicina

La burocratización de los

sistemas de salud aporta

su responsabilidad a la

deshumanización cuando una

organización asistencial está

basada solamente en la rapidez

y en la eficacia siente que fracasa.

La burocratización de los sistemas de salud aporta su responsabilidad a la deshumanización cuando una organización asistencial está basada solamente en la rapidez y en la eficacia y no motiva a los profesionales a superarse, sino a sostenerse en una rutina estructurada.

La rigidez administrativa de algunas instituciones de salud imprimen un elemento extra de preocupación al enfermo, dificultando el acceso directo a los servicios en general y a los profesionales en particular, dilatando turnos y no facilitando la resolución inmediata de cuestiones no médicas.

\section{Impacto de la aparatología en la asistencia sanitaria}

Los científicos desarrollaron una importante actividad desde mediados del siglo XX, cuya investigación devino en la implementación de nueva aparatología en el servicio hospitalario. 
Este hecho modificó positivamente la evolución y pronóstico de muchas patologías, pero lamentablemente conllevó, por su inadecuada manipulación en ocasiones, a la despersonalización de la asistencia, junto con otros factores.

A los fines de este trabajo, se citan dos invenciones en particular consideradas paradigmáticas para la bioética: la hemodiálisis y la asistencia respiratoria mecánica.

Equipos para hemodiálisis: fue en 1960 en Seattle, donde se dispuso por primera vez de un acceso vascular permanente para hemodiálisis periódicas ("shunt de teflón") desarrollado por Dr. Belding Scribner ${ }^{4}$ y Wayne Quinton ${ }^{5}$ (Álvarez, 1996).

Este trascendente invento marcó un hito en la historia de la nefrología y en la supervivencia de las personas con insuficiencia renal crónica, generando el desafío ético referente a quién debía tomar la decisión sobre la elección de enfermos a tratar.

\section{Intervención bioética}

Todos los pacientes tenían la indicación médica de diálisis, pero el número superaba la cantidad de equipos disponibles. Esto provocó un dilema entre los principios bioéticos de beneficencia y justicia (Beauchamp y Childress, 1979).

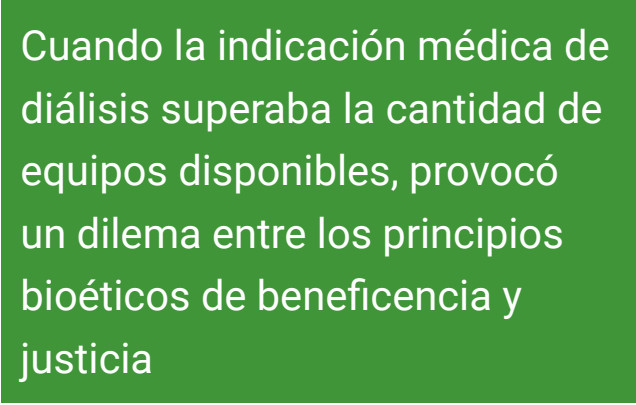

Cuando la indicación médica de diálisis superaba la cantidad de equipos disponibles, provocó un dilema entre los principios justicia
Por lo anteriormente dicho, se decidió conformar un comité integrado por profesionales y representantes de la comunidad para que analizaran cada caso y establecieran criterios no médicos para incluir o excluir pacientes.

Este evento se considera históricamente como el primer comité donde no solo participaron profesionales de la salud. Su repercusión fue tal, que motivó la publicación de un artículo en la revista Life en 1962, escrito por la periodista Shana Alexander titulado "They decide: Who Lives, Who Dies".

Equipos de asistencia respiratoria mecánica (ARM): el primer respirador eléctrico a presión negativa y popularmente conocido como "pulmón de acero", fue desarrollado por Drinker ${ }^{6}$ y Shaw ${ }^{7}$ en 1928.

Luego, en 1931, Emerson ${ }^{8}$ logró mejorarlo, incorporando la presión positiva. Finalmente, en las décadas de los 50 y 60 continuaron los perfeccionamientos en el diseño que condujeron a los modernos respiradores microprocesados que conocemos hoy (Rodríguez, 2012).

4 Belding Scribner (Chicago 1921 - Seattle 2003), médico estadounidense pionero en tratamientos de diálisis.

5 Wayne Quinton (1921-2015), considerado el padre de la bioingeniería.

6 Philip Drinker (1894-1972), ingeniero químico. Su hermano Cecil, era fisiólogo en la Universidad de Harvard.

7 Louis Agassiz Shaw Jr. (25 de septiembre de 1886 - 27 de agosto de 1940), instructor de fisiología en la Escuela de Salud Pública de la Universidad de Harvard.

8 John Haven Emerson (1906-1997), inventor americano especialista en equipamientos médicos, especialmente para uso respiratorio. 
Por su complejidad debieron ser implementados en las unidades de cuidados críticos; siendo de gran utilidad en los casos agudos, pero en los crónicos se transforman en fútiles; prolongando la vida de pacientes sin posibilidades de recuperación por la patología subyacente y generando dilemas en torno a la retirada de ellos.

Sumado a lo anterior, en 1967, el primer trasplante cardíaco efectuado en Sudáfrica por el Dr. Christian Barnard, concibió un cambio en el concepto de muerte por parada cardíaca y respiratoria vigente hasta ese momento, por el de muerte cerebral.

\section{Intervención bioética}

El cambio en el concepto de

muerte obliga a reflexionar

sobre los principios bioéticos de

autonomía, beneficencia,

no-maleficencia y justicia
La situación planteada no vela por el respeto del valor de la vida digna de las personas en su etapa final y obliga a reflexionar sobre los principios bioéticos de autonomía, beneficencia, nomaleficencia y justicia.

Por este motivo, en el año 1968, fue necesario convocar a un comité interdisciplinario presidido por el Dr. Henry Beecher en Harvard, cuyo trabajo concluyó en la elaboración de un documento en referencia a los criterios de muerte cerebral y a las posibilidades jurídicas y éticas de desconexión de ARM.

De ello se desprendió el consecuente beneficio de preservación de los órganos para su ulterior trasplante. Este evento es considerado como el primer comité de bioética hospitalario (citado por Gherardi, 2008).

\section{Aportes de la Orden Hospitalaria de San Juan de Dios a la humanización de la salud}

La Orden Hospitalaria de San Juan de Dios ${ }^{9}$ establece en sus Constituciones ${ }^{10}$ que ese nombre expresa su identidad y la razón de su existencia en la Iglesia, vivida y manifestada a través del carisma de la hospitalidad al estilo de su fundador, san Juan de Dios.

Una orden religiosa cuyo origen se remonta al siglo XVI, que nace del Evangelio de la Misericordia y centra su carisma en la hospitalidad, se mantiene vigente en 51 países de los cinco continentes, enraizada en valores guía tales como el respeto, la calidad, la responsabilidad y la espiritualidad ${ }^{11}$.

La propia orden los define de la siguiente manera:

Respeto: por el prójimo, humanización, dimensión humana, responsabilidad recíproca para con los Colaboradores y Hermanos, comprensión, visión holística,

9 Aprobada por el papa san Pío V, el 1 de enero de 1572.

10 Constituciones de la Orden Hospitalaria de San Juan de Dios. Acta fundacional. Const. 1585, intr.

11 Definiciones disponibles en el sitio web oficial www.ohsjd.org 
promoción de la justicia social, derechos civiles y humanos, implicación de los familiares.

Calidad: excelencia, profesionalidad, atención holística, conciencia de las nuevas necesidades, modelo de unión con nuestros Colaboradores, modelo de atención juandediana, arquitectura y decorado acogedor, colaboración con terceros.

\section{El centro de interés de cuantos \\ vivimos y trabajamos en \\ el hospital o en otra obra \\ asistencial es la persona \\ asistida}

Responsabilidad: fidelidad a los ideales de San Juan de Dios y de la Orden, ética (bioética, ética social, ética de gestión) protección del medio ambiente, responsabilidad social (para los europeos: la Unión Europea), sostenibilidad, justicia, justa distribución de los recursos.

Espiritualidad: pastoral de la salud, evangelización, ofrecer atención espiritual a los miembros de otras religiones, ecumenismo, colaboración con parroquias, diócesis, otras confesiones religiosas.

En el año 2000, una comisión ad hoc elaboró un documento especial denominado Carta de Identidad, con motivo de la celebración en Granada del LXV Capítulo General de la Orden; en la misma se abordan los diferentes aspectos que hacen a la vida de las instituciones juandedianas y se constituyó como elemento de consulta imprescindible para iluminar la hospitalidad.

En el capítulo 1.1. refiere al número 43 de sus Estatutos Generales ${ }^{12}$, que en otros importantes argumentos establece:

a) El centro de interés de cuantos vivimos y trabajamos en el hospital o en otra obra asistencial es la persona asistida.

b) Promovemos y defendemos los derechos del enfermo y necesitado, teniendo en cuenta su dignidad personal.

c) Nos comprometemos decididamente en la defensa y promoción de la vida humana.

d) Reconocemos el derecho de las personas asistidas a ser convenientemente informadas de su estado de salud.

e) Observamos las exigencias del secreto profesional y tratamos de que sean respetadas por cuantos se relacionan con los enfermos y necesitados.

f) Defendemos el derecho a morir con dignidad y a que se respeten y atiendan los justos deseos y las necesidades espirituales de quienes están en trance de muerte, sabiendo que la vida humana tiene un término temporal y que está llamada a la plenitud en Cristo.

El citado documento ${ }^{13}$ consta de ocho capítulos, con temas eje para la actividad asistencial de los centros asistenciales de la orden, a saber:

I. Principios, carisma y misión de la Orden Hospitalaria de San Juan de Dios.

12 Estatutos Generales de la Orden Hospitalaria, pág. 25.

13 Carta de Identidad de la Orden Hospitalaria de San Juan de Dios. Año 2000. 
II. Fundamentos bíblico-teológicos de la hospitalidad.

III. El carisma de la hospitalidad en San Juan de Dios y en la orden hospitalaria.

IV. Principios que iluminan nuestra hospitalidad.

V. Aplicación a situaciones concretas.

VI. Formación, docencia e investigación.

VII. Integridad personal como base para la acción.

VIII. Crear el futuro con esperanza.

Una asistencia integral de

excelencia será la efectuada por

personas idóneas y centrada en

las personas asistidas
Especialmente, el capítulo IV hace referencia al respeto por la vida y la dignidad de la persona humana como bien fundamental, promoviendo la protección de las vulnerabilidades y velando por el sostenimiento de los recursos naturales.

El capítulo V, dedicado a la aplicación a situaciones concretas, contempla ampliamente la práctica de la bioética global.

Esta herramienta fundamental para la labor asistencial diaria también aplica en la gestión y en la dirección de los centros, en las políticas económicas y financieras, en las políticas de recursos humanos, medioambientales y de responsabilidad social.

1) Es impensado desconocer la trascendencia que los adelantos científicos y tecnológicos imprimieron desde mediados del siglo pasado a la medicina. Estos beneficios mejoraron la calidad y la expectativa de vida optimizando la actividad asistencial.

2) Del mismo modo es arbitrario sostener que todo fue beneficioso y desconocer que su implementación en ocasiones generó problemáticas, que confrontaron a los equipos de salud, pacientes y familiares con dilemas éticos previamente inexistentes.

3) En referencia al impacto de las tecnologías sobre la deshumanización, esta se produce cuando la preocupación se centra más en la operatividad del aparato y no se priorizan las necesidades del enfermo.

Finalmente, una asistencia integral de excelencia será la efectuada por personas idóneas y centrada en las personas asistidas; donde las tecnologías brinden un aporte complementario y no reemplacen por ninguna circunstancia la atención personalizada, con calidad, respeto, espiritualidad y responsabilidad: principios base de la hospitalidad, al estilo de san Juan de Dios. 


\section{Bibliografía}

Abel, F. (2001). Bioética, orígenes, presente y futuro. Instituto Borja de Bioética. Fundación Mapfre Medicina. Madrid: Ed Mapfre. Citado por Cambá F. J. Comités de Bioética. Bibliografía del Máster IBB-URLL. 2011, 9. ${ }^{a}$ edición.

Ad Hoc Committee of the Harvard Medical School to examine the definition of brain death (1968). A definition of irreversible coma. JAMA, (205), 337-40. Citado por Gherardi, C. (2008). Revista Medicina Buenos Aires, 68(5).

Alexander, S. (1962). They Decide Who Lives, Who Dies. Life, (53), 102-125. Recuperado de https://books.google.com.ar/books?id=qUoEAAAAMBAJ\&pg=PA4\&lpg=PA4\&dq=Life+53+1962+november+9\&source=b I\&ots=9bJofii298\&sig=Q1Zv25jruyn5rwuFNu3YHoyr7tM\&hl=es\&sa=X\&ved=0ahUKEwib6r_NvK_ZAhVLj ZAKHSYbAvwQ6AEIKDAA\#v=onepage\&q=Life\%2053\%201962\%20november\%209\&f=false

Álvarez, F. (1996). Hemodiálisis: Evolución histórica y consideraciones generales. Revista de Nefrología, 16(4), 25-36. Recuperado de http://www.revistanefrologia.com/es-publicacion-nefrologia--articulo-hemodialisis-evolucion-historica-consideraciones-generales-X0211699596023466

Marchesi, P., Redrado, J. L., Brusco A., Gol Gurina, J., Bolech, P. (1988). Humanización en salud. Bogotá: Colección SELARE.

Marchesi, P. (1988). Por un hospital más humano. Bogotá: Colección SELARE.

Martínez Gil, J. L. (2010). Superiores Generales, Santos y Beatos de la Orden de San Juan de Dios, 1536-2010. Madrid: Ed. Hospitalaria. 29-438-440.

Orden hospitalaria San Juan de Dios. (2012). Carta de Identidad de la Orden Hospitalaria de San Juan de Dios. Madrid: Fundación Juan Ciudad.

Ordi, J. (2011). La persona, una estructura pluridimensional. Bibliografía del Máster IBB-URLL. 9. ${ }^{\text {a }}$ edición. 1-7.

Rodríguez, A. (2012). Historia de la ventilación mecánica. Revista Medicina Intensiva Argentina, 29(1). Recuperado de http://revista.sati.org.ar/index.php/MI/article/viewFile/302/251 